\title{
Raman Spectra of Hydrogen Peroxide in Condensed Phases. I. The Spectra of the Pure Liquid and Its Aqueous Solutions*
}

\author{
RoBert C. TAYLOR, Department of Chemistry, University of Michigan, Ann Arbor, Michigan \\ AND \\ PaUl C. CRoss, Department of Chemistry, University of Washington, Seattle, Washington
}

(Received April 14, 1955)

\begin{abstract}
The Raman spectrum of liquid $99.5 \% \mathrm{H}_{2} \mathrm{O}_{2}$ has been obtained at $30^{\circ}$ and $-40^{\circ} \mathrm{C}$ and compared with the spectrum of $90 \% \mathrm{D}_{2} \mathrm{O}_{2}$ at $-40^{\circ} \mathrm{C}$. Three bands not previously reported were observed and their assignment, together with that of the remaining bands, is discussed.

Raman spectra were also obtained for aqueous solutions at $30^{\circ}$ and $-40^{\circ} \mathrm{C}$ over the complete concentration range. The interpretation is qualitatively in agreement with the results of other work which indicate that the addition of hydrogen peroxide to water results in more extensive hydrogen bonding and an increased degree of order in the solutions.
\end{abstract}

\section{INTRODUCTION}

$\mathrm{H}$ YDROGEN peroxide is a simple compound which is strongly hydrogen bonded in its condensed phases. Its vibrational spectrum under such conditions is therefore not only of interest in the study of the molecule but also in the comparative study of associated liquids. The infrared spectrum of the liquid has been reported fairly recently ${ }^{1-3}$ but apparently the Raman spectrum has not been examined since the work of Simon and Feher ${ }^{4,5}$ over fifteen years ago. These workers were primarily interested in locating the fundamental frequencies of the molecule and did not concern themselves with what may be termed intermolecular effects nor with the effect of temperature on the appearance of the spectrum.

In the case of water, which is the liquid most closely resembling hydrogen peroxide, the Raman spectrum exhibits several bands ${ }^{6,7}$ which cannot be explained in terms of the fundamental frequencies of the molecule. These have been attributed to intermolecular motions in the quasi-crystalline lattice maintained in the liquid by the hydrogen bonds between the individual molecules. Observation of similar frequencies in hydrogen bonded liquids other than water is of interest since they afford direct evidence regarding the freedom of motion of the individual molecules. Such bands have not previously been reported in the case of hydrogen peroxide.

\section{EXPERIMENTAL}

Hydrogen peroxide containing approximately $10 \%$ by weight of water was kindly donated by the Buffalo Electrochemical Company. It was concentrated in the

*The initial stages of this work were carried out at Brown University under contract N6ori-88 with the Office of Naval Research.

1 P. A. Giguere, J. Chem. Phys. 18, 88 (1950).

2 R. C. Taylor, J. Chem. Phys. 18, 898 (1950)

${ }^{3} \mathrm{O}$. Bain and P. A. Giguere, Can. J. Chem. 33, 527 (1955).

4 A. Simon and F. Feher, Z. Elektrochem. 41, 290 (1935).

$5 \mathrm{~F}$. Feher, Ber. deut chem. Ges. 72B, 1778 (1939).

6 J. H. Hibben, J. Chem. Phys. 5, 166 (1937).

7 Cross, Burnham, and Leighton, J. Am. Chem. Soc. 59, 1134 (1937). manner described by Gross and Taylor ${ }^{8}$ to give material containing $0.5 \%$ or less of water. Solutions were prepared by dilution of this material with special highpurity conductivity water. In all cases it was found essential to evaporate the sample in vacuo directly into the pretreated Raman cell just prior to an exposure. If this was not done, trace decomposition under the arcs invariably produced bubbles which reflected stray light into the spectrograph and reduced the quality of the spectrum. Deuterium peroxide was prepared by persulfate oxidation of heavy water in a closed system ${ }^{9}$; the most concentrated solution examined contained about $90 \% \mathrm{D}_{2} \mathrm{O}_{2}$ and $10 \% \mathrm{D}_{2} \mathrm{O}$.

Spectra of the pure peroxide were obtained with a Gaerntner two prism glass spectrograph with a $f / 3.5$ camera lens while the solution spectra, which were obtained in the early stages of the investigation, were photographed with a medium Hilger spectrograph with glass optics and an aperture of about $f / 10$. The dispersion of the first instrument is about 38 angstroms per $\mathrm{mm}$ in the blue region and that of the latter about 13 angstroms per mm. Type AH-11 mercury arcs were used in the light source and the incident light was heavily filtered with $\mathrm{NaNO}_{2}$ and Rhodamine 5GDN Extra solutions so as to give as clean spectra as possible. Temperature control of the sample was achieved by placing the

TABLE I. Raman bands of liquid hydrogen and deuterium peroxides.

\begin{tabular}{|c|c|c|c|c|}
\hline \multicolumn{2}{|c|}{$\mathrm{H}_{2} \mathrm{O}_{2}$, liq. } & \multirow{2}{*}{$\begin{array}{c}\mathrm{D}_{2} \mathrm{O}_{2} \text {, liq. } \\
(90 \%) \\
-40^{\circ} \mathrm{C}\end{array}$} & \multirow[b]{2}{*}{ Assignments } & \multirow[b]{2}{*}{ Polariz. } \\
\hline $30^{\circ} \mathrm{C}$ & $-40^{\circ} \mathrm{C}$ & & & \\
\hline$\ldots$ & $200 \pm 25 \mathrm{~cm}^{-1}$ & $\cdots$ & & \\
\hline$\ddot{380 \pm 0.5}$ & $\begin{array}{l}525 \pm 25 \\
880 \pm 0.5\end{array}$ & $880+0.5$ & $\nu_{a}(\mathrm{a})$ & pol. \\
\hline $1400 \pm 5$ & $1402 \pm 5$ & $1013 \pm 5$ & $\nu_{2}(\mathrm{a})$ & pol. \\
\hline$\ldots$ & $2815 \pm 15$ & $2038 \pm 10$ & $2 \nu_{2}$ (A) & $\cdots$ \\
\hline $3410 \pm 8$ & $3364 \pm 8$ & $2472 \pm 7$ & $\nu_{1}(\mathrm{a})$ & pol. \\
\hline
\end{tabular}

${ }^{8}$ P. M. Gross, Jr. and R. C. Taylor, J. Am. Chem. Soc. 72, 2075 (1950).

${ }_{9}^{2}$ F. Feher, Ber. deut. chem. Ges. 72, 1789 (1939). 


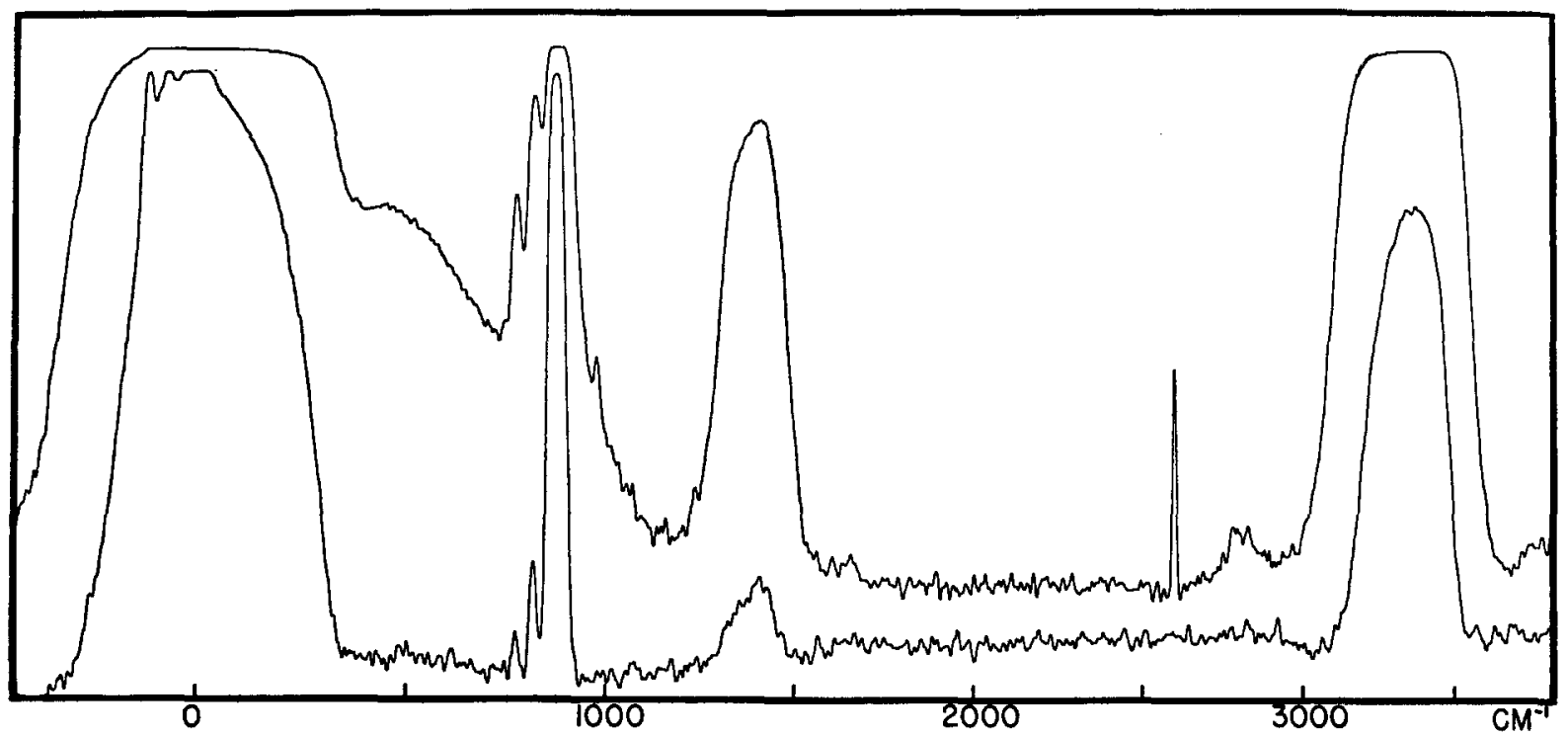

FIg. 1. The Raman spectrum of liquid hydrogen peroxide at $-40^{\circ} \mathrm{C}$ (short and long exposures).

Raman cell in a small Dewar flask with windows in the bottom and passing air of the desired temperature up around it. The temperature gradient along the cell was approximately 5-7 degrees.

\section{EXPERIMENTAL RESULTS}

The values measured for the band maxima in the spectra of pure $\mathrm{H}_{2} \mathrm{O}_{2}$ and $90 \% \mathrm{D}_{2} \mathrm{O}_{2}$ are tabulated in Table I. Since all the bands except one were broad and rather diffuse, most of the measurements were made on microphotometer tracings. The uncertainties listed in the table consequently reflect primarily the difficulty in choosing the maxima. Microphotometer tracings of the Raman spectrum of $99.5 \% \mathrm{H}_{2} \mathrm{O}_{2}$ at $-40^{\circ} \mathrm{C}$ are shown in Fig. 1 for both long and short exposures.

In the case of peroxide-water mixtures, significant changes occurred in the shape of the $\mathrm{O}-\mathrm{H}$ stretching band but otherwise, the spectra of the solutions are satisfactorily interpreted in terms of the spectra of the pure compounds. The systematic changes in the shape of the $\mathrm{OH}$ band as water is added to hydrogen peroxide are shown in Fig. 2. Table II lists the measured maxima of the bands arising from hydrogen motions in solutions at $-40^{\circ} \mathrm{C}$. The exposures in the case of the solutions

TABLE II. Raman bands arising from hydrogen motions in mixtures of water and hydrogen peroxide at $-40^{\circ} \mathrm{C}$.

\begin{tabular}{|c|c|c|c|c|}
\hline \multirow{2}{*}{$\begin{array}{l}\mathrm{Mol} \text { ratio } \\
\mathrm{H}_{2} \mathrm{O}_{2}: \mathrm{H}_{2} \mathrm{O}\end{array}$} & \multicolumn{2}{|c|}{ Bending motions } & \multicolumn{2}{|c|}{ Stretching motions } \\
\hline & $\mathrm{H}_{2} \mathrm{O}_{2}$ & $\mathrm{H}_{2} \mathrm{O}$ & Shoulder & Main max \\
\hline $\begin{array}{l}9: 2 \\
2: 1 \\
1: 1 \\
1: 2 \\
1: 4^{\mathrm{a}} \\
1: 9^{\mathrm{b}}\end{array}$ & $\begin{array}{l}1411 \mathrm{~cm}^{-1} \\
1422 \\
1431 \\
1465 \\
1455 \\
1445\end{array}$ & $\begin{array}{l}\cdots \\
1625 \\
1630 \\
1638 \\
1640 \\
1630\end{array}$ & $\begin{array}{c}\cdots \\
\cdots \\
3260 \pm 20 \\
3240 \pm 20 \\
3345 \pm 20\end{array}$ & $\begin{array}{l}3373 \mathrm{~cm}^{-1} \\
3377 \\
3395 \\
3412 \\
3417 \\
3417\end{array}$ \\
\hline
\end{tabular}

$-25^{\circ} \mathrm{C}$.

b $-8^{\circ} \mathrm{C}$ were not sufficiently long to allow reliable measurements to be made of the weaker bands observed in the pure peroxide spectrum. The $\mathrm{O}-\mathrm{O}$ stretching band of hydrogen peroxide at $880 \mathrm{~cm}^{-1}$ was not shifted in any of the solutions.

\section{DISCUSSION}

The bands in the spectrum of pure hydrogen peroxide at 880,1400 , and $3410 \mathrm{~cm}^{-1}$ have been reported previously ${ }^{4}$ as have also the corresponding bands of the deuterated compound. ${ }^{5}$ On the basis of the $\mathrm{C}_{2}$ model commonly accepted for this molecule, the six normal vibrations may be described as a symmetrical and unsymmetrical hydrogen bending motion, a symmetrical and unsymmetrical hydrogen stretching motion, an oxygen-oxygen stretching motion, and a torsional motion of the $\mathrm{OH}$ groups around the $\mathrm{O}-\mathrm{O}$ axis. Feher ${ }^{5}$ has suggested, and the evidence seems to support the fact, that because of the particular geometry of the molecule the two hydrogen bending motions, $\nu_{2}$ and $\nu_{6}$, have very nearly the same frequency, and likewise in the case of the two hydrogen stretching motions, $\nu_{1}$ and $\nu_{5}$. Although Simon and Feher originally reported ${ }^{4}$ the 1400 $\mathrm{cm}^{-1}$ band split into a close doublet, this phenomenon was not found in their deuterium work ${ }^{5}$ and also was not observed in the present investigation. Since the 1400 $\mathrm{cm}^{-1}$ band was observed to be fairly strongly polarized, it appears to be due largely to the symmetrical bending motion, $\nu_{2}$. The microphotometer tracing shows a shoulder on the low-frequency side of this band at approximately the position due to the unsymmetrical bending motion which one would normally expect to be more intense in the infrared. In the Raman spectrum of the crystal, these two bands are clearly resolved and sharp..$^{10}$

${ }^{10}$ R. C. Taylor (unpublished work). 
In a similar way, the high degree of polarization of the $3410 \mathrm{~cm}^{-1}$ band indicates that it must be ascribed chiefly to the symmetrical hydrogen stretching motion, $\nu_{1}$. In this case the position of the infrared band coincides with that of the Raman band and no asymmetry is apparent, although the accidental degeneracy again is removed in the crystal and both bands are well resolved in the latter's Raman spectrum. ${ }^{10}$ No ambiguity exists in the assignment of the $880 \mathrm{~cm}^{-1}$ band to the $\mathrm{O}-\mathrm{O}$ stretching motion.

The remaining three bands at 200,525 , and $2815 \mathrm{~cm}^{-1}$ in the Raman spectrum of pure peroxide have not been previously reported. The infrared spectra of both the liquid and vapor show a band in the general region of $2700 \mathrm{~cm}^{-1}$ whose assignment has not been clearly established. Deuterium studies show that it is primarily a hydrogen motion and the simplest explanation of this infrared band is that it is a combination band of the two bending modes, $\nu_{2}$ and $\nu_{6}$, although there is some discrepancy in the calculated and observed positions. On the other hand, the Raman band in the liquid at 2815 $\mathrm{cm}^{-1}$ would appear more likely to be the overtone of $\nu_{2}$, the symmetrical bending motion, with perhaps some contribution from $2 \nu_{6}$ which belongs to the same class. In fact, the small discrepancy between the observed and calculated positions of this band may be due to a weak Fermi resonance between the two overtones acting to increase the frequency of $2 \nu_{2}$. This band was observed excited by both the $e$ and $k$ lines of the mercury arc.

The bands observed at approximately 200 and 525 $\mathrm{cm}^{-1}$ in the Raman spectrum fall in the range normally associated with intermolecular frequencies of compounds with light atoms. The Raman spectrum of water, for example, contains two broad and diffuse bands centered roughly around 200 and $600 \mathrm{~cm}^{-1}$ which have been attributed respectively to hindered translational and rotational motions. ${ }^{7}$ The least moment of inertia of the hydrogen peroxide molecule around an axis nearly coincident with the $\mathrm{O}-\mathrm{O}$ axis is the same order of magnitude as the moments of inertia of the water molecule. Inasmuch as the intermolecular forces in the liquid must be very similar to those in liquid water, a band in the general region of $500 \mathrm{~cm}^{-1}$ might be predicted. Also, the two halves of the peroxide molecule may execute a torsional motion around the $\mathrm{O}-\mathrm{O}$ axis, the frequency of which has been predicted theoretically to lie in this same region. Since this internal torsional motion would be very strongly coupled to any restricted rotational motion around the same axis, it seems probable that the scattering in the region between 400 and 700 $\mathrm{cm}^{-1}$ cannot be attributed solely to any simple type of vibration.

The low-frequency scattering lying between the exciting line and about $300 \mathrm{~cm}^{-1}$ is fairly intense and can be assigned either to restricted rotational motions involving the larger motions of inertia or to translatory oscillations, or both. The Raman spectrum of the crystal $^{10}$ shows at least seven well-defined lines in this
Fig. 2. The $\mathrm{O}-\mathrm{H}$ stretching band in hydrogen peroxide-water solutions at $-40^{\circ} \mathrm{C}$ for various values of the mole ratio $\mathrm{H}_{2} \mathrm{O}_{2}: \mathrm{H}_{2} \mathrm{O}$.

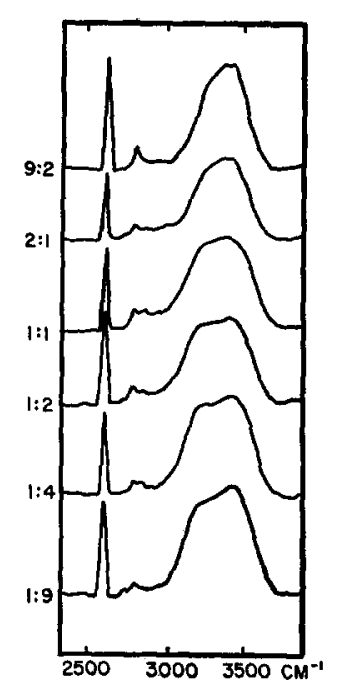

region, some of them approaching the $880 \mathrm{~cm}^{-1}$ line in intensity, which must involve both rotatory and translatory types of lattice vibrations. Apparently the hydrogen bonding present in liquid is sufficient to maintain some sort of a three-dimensional quasi-lattice network in which these vibrations can occur in strongly perturbed forms. Unfortunately, the $\mathrm{D}_{2} \mathrm{O}_{2}$ spectra were not of sufficient quality to allow the position of these rather diffuse bands to be distinguished with any certainty.

There are two points of interest in the spectra of the aqueous solutions, both consistent with the same general conclusion. As hydrogen peroxide is added to water, the shoulder on the low-frequency side of the $\mathrm{O}-\mathrm{H}$ stretching band progressively increases in intensity until it becomes a distinct maximum in the concentration range of approximately twenty to forty mole percent hydrogen peroxide (Fig. 2); at a temperature of $30^{\circ} \mathrm{C}$ the shoulder increases in intensity but does not reach a maximum. A discussion of the origin of this second component of the $\mathrm{OH}$ band will not be given here. Instead it will merely be pointed out that its intensity has been shown empirically to correlate very well with the extent to which hydrogen bonding occurs in the liquid. ${ }^{11}$

Secondly, inspection of Table II shows that the positions of both the $1400 \mathrm{~cm}^{-1}$ band of $\mathrm{H}_{2} \mathrm{O}_{2}$ and the 1630 $\mathrm{cm}^{-1}$ band of water shift with concentration, the highest frequencies for both bands being observed in the same concentration range mentioned above. It is generally accepted that an increase in the amount of hydrogen bonding in a liquid shifts the position of hydrogen bending bands such as these to higher frequencies. The spectral data presented here thus are in good agreement with the results of freezing point measurements, ${ }^{12}$ dielectric constant measurements ${ }^{8}$ and thermodynamic

11 See, for example, reference 6 and also: J. Burnham and P.A. Leighton, J. Am. Chem. Soc. 59, 424 (1937); J. Chien, J. Am. Chem. Soc. 69, 20 (1947), and others.

${ }^{12}$ W. T. Foley and P. A. Giguere, Can. J. Chem. 29, 123 (1951). 
R. C. TAYLOR AND P. C. CROSS

data ${ }^{13}$ all of which indicate that the addition of hydrogen peroxide to water in concentrations up to around 40 mole percent results in an increase in the number of hydrogen bonds and a greater degree of order in the solution.

${ }^{13}$ A. G. Mitchell and W. F. K. Wynne-Jones, Discussions Faraday Soc. No. 15, 161 (1953).

\section{ACKNOWLEDGMENTS}

One of the authors (R.C.T.) is indebted to the National Research Council for the grant of a predoctoral fellowship during the early portion of the work.

Note added in proof.-Simon and Kriegsmann, Naturwissenschaften 42,12 (1955) have recently reported a band at $228 \mathrm{~cm}^{-1}$ in the Raman spectrum of the liquid at $5^{\circ} \mathrm{C}$.

THE JOURNAL OF CHEMICAL PHYSICS

VOLUME 24, NUMBER 1

J A N U A R Y, 1956

\title{
Influence of Fermi Resonance on the Centrifugal Stretching Constant of a Linear Molecule*
}

\author{
Gilbert Amat, $\dagger$ Mark Goldsmtit, and Harald H. Nielsen \\ Department of Physics and Astronomy, The Ohio State University, Columbus, Ohio
}

(Received March 28, 1955)

\begin{abstract}
A theory of the influence of Fermi resonance on the centrifugal distortion constant of a linear molecule has been developed and its predictions for $\mathrm{CO}_{2}$ compared with experiment. The theory of those bands which exhibit simultaneous Fermi resonance and $l$-type doubling is also discussed.
\end{abstract}

\section{INTRODUCTION}

A GENERAL quantum-mechanical study of the rotation-vibration energy levels of molecules has previously been given, ${ }^{\mathbf{1}}$ but as is well known, this theory is essentially a nondegenerate perturbation analysis which must be modified to cope with accidental degeneracies. One of the most interesting theoretically, and commonly encountered experimentally, of these degeneracies occurs when a molecule possesses a normal mode of vibration whose fundamental frequency is approximately equal to one half the frequency of one of the other normal modes. If, in addition to this condition, the eigenfunctions of two almost equal vibrational energy levels belong to the same symmetry species, the vibrational interaction known as Fermi resonance occurs. In the present paper we examine the effects of this interaction on the rotational energy levels of a linear molecule.

Let $V_{1}, V_{2}, \cdots, V_{8}$ be the principal vibrational quantum number associated with the fundamental frequencies $\omega_{1}, \omega_{2}, \cdots, \omega_{s}$ of a linear molecule. We suppose that $\omega_{1} \approx 2 \omega_{2}$; that the corresponding wave functions are of the same symmetry species, and that $\omega_{1}$ is nondegenerate whereas $\omega_{2}$ may be either degenerate or nondegenerate. Under these conditions, the vibrational energy levels of the molecule fall into resonance polyads ${ }^{1}$ whose components have approximately equal energies. The levels of a polyad are characterized by some fixed

* This work was supported in part by Contract N6-onr.22526 NR 019-403, with the Office of Naval Research and by contract DA-33-019-ORD-1507, with the Office of Ordnance Research.

† Foreign Operations Administration Fellow (1954-1955) on leave from the Laboratoire d'Infrarouge P.C.B., Paris.

${ }_{1}$ H. H. Nielsen, Phys. Rev. 68, 181 (1945); Revs. Modern Phys. 23, 90 (1951). value of $2 V_{1}+V_{2}$ and, if $\omega_{2}$ is degenerate, a value of $l_{2}$, the quantum number of angular momentum associated with $\omega_{2}$. In the presence of first-order anharmonic resonance interaction (Fermi resonance), the vibrational energies $X_{k}$ of the members of a given polyad may be obtained as roots of the secular equation:

$$
\left|\begin{array}{cccc}
E_{V 1}-X & W_{12} & 0 & \cdots \\
W_{21} & E_{V^{2}}-X & W_{23} & \cdots \\
0 & W_{32} & E_{V 3}-X & \cdots \\
\cdot & \cdot & \cdot & \\
\cdot & \cdot & \cdot & \\
\cdot & \cdot & \cdot
\end{array}\right|=0
$$

where $E_{V 1}, E_{V 2}, \cdots$ designate the unperturbed vibrational energies given by:

$$
\begin{aligned}
E_{V} / h c & =\sum_{s}\left(V_{s}+g_{s} / 2\right) \omega_{s} \\
& +\sum_{s s^{\prime}} x_{s s^{\prime}}\left(V_{s}+\frac{g_{s}}{2}\right)\left(V_{s^{\prime}}+\frac{g_{s}^{\prime}}{2}\right)+\sum_{s} x_{s} l_{s} l_{s}{ }^{2},
\end{aligned}
$$

$g_{s}$ being the degree of degeneracy of $\omega_{s}$ and where the $W_{i, i \pm 1}$ are the matrix elements:

$$
\left(V_{1} V_{2}\left|h c K_{122} q_{1} q_{2}^{2}\right| V_{1} \mp 1, V_{2} \pm 2\right)
$$

in which $K_{122}$ is the coefficient of a cubic term in the potential energy. If $\omega_{2}$ is twofold degenerate, as will be true in our calculations, one finds ${ }^{1}$ :

$$
\begin{aligned}
\left(V_{1} V_{2}\left|h c K_{122} q_{1} q_{2}{ }^{2}\right| V_{1}\right. & \left.-1, V_{2}+2\right) \\
& =-\frac{h c K_{122}}{2}\left[\left(V_{2}+2\right)^{2}-l_{2}{ }^{2}\right]\left(\frac{V_{1}}{2}\right)^{\frac{1}{2}} .
\end{aligned}
$$

